Chattaway, F. W. \& Barlow, A. J. E. (1954). J. gen. Microbiol. 11, 506-511.

\title{
The Fluorescent Materials produced in vivo by certain Dermatophytes
}

\author{
By F. W. CHATTAWAY \\ Department of Biochemistry, University of Leeds \\ AND A. J. E. BARLOW \\ Department of Dermatology, Royal Infirmary, Huddersfield
}

SUMMARY: The fluorescent materials present in human and cat hair as a result of infection with Microsporon canis, M. audouini and Trichophyton schoenleini were extracted with dilute ammonia. Paper chromatography and electrophoresis showed that the principal fluorescent material was common to all these infections and that further fluorescent substances were present in the extract from $T$. schoenleini infected hair. Chemical properties and absorption spectra of the extracts have been examined.

The brilliant green fluorescence in Wood's light shown by hairs infected with Microsporon canis, $M$. audouini and Trichophyton schoenleini was first noticed by Margarot \& Devèze (1925) and is now an accepted method of diagnosis in cases of small-spore ringworm, although the nature of the fluorescent material has not been determined. Davidson \& Gregory (1932) showed that it could be readily extracted from the hairs by hot water and, working with a crude extract, they showed the presence of a substance or substances containing nitrogen, phenolic and aldehyde groups but no ninhydrin-reacting substances. Felsher (1949) extracted the hairs with cold $2 \mathrm{~N}$-sodium bromide and showed colour changes of the fluorescence with $\mathrm{pH}$, and quenching by a number of salt solutions. Robinson, Figge \& Bereston (1953) described spectroscopic studies made on an aqueous extract of hair infected with Microsporon audouini; they purified their extract by shaking with a mixture of alumina, aluminium silicate and talc under unspecified conditions. Infra-red spectra showed the presence of material containing a substituted amide group, hydroxyl, methyl and amino groups. The present work describes a comparison of the fluorescent substances produced by the three fungi, and indicates certain common products and the presence of additional fluorescent materials in the extract from the Trichophyton schoenleini infection. Isolation of the pure substances has not been achieved, principally owing to the very small amounts available.

\section{MATERIALS AND METHODS}

Infected hair. This was obtained from patients in Leeds and Huddersfield and also in the case of infections due to Microsporon canis from the hair of cats infected with Microsporon. Hair was also obtained from a case of favus which occurred in an Italian immigrant. Mycological identification of the fungus was made in all cases.

Extraction of the fluorescent material. Infected hair was extracted with ether 
for $24 \mathrm{hr}$. in a Soxhlet extractor to remove fatty materials. Boiling with $1 \cdot 17 \mathrm{~N}-\mathrm{NH}_{3}$ for $20 \mathrm{~min}$. gave complete extraction of the fluorescent substance. This method was preferred to extraction for $1 \mathrm{hr}$. with boiling water as employed in the other studies cited, since this was not always found to give complete extraction. The ammoniacal extract was immediately concentrated to dryness in vacuo at $40^{\circ}$. The material was maintained in the dry state at $-20^{\circ}$ since it was found that fluorescence faded steadily on keeping in aqueous solution.

Chromatography. The method of Consden, Gordon \& Martin (1944) was used with Whatman papers nos. 3 and 4 previously washed with water and equilibrated in the presence of the solvent mixture before development of the chromatogram. The solvent mixtures which proved of the greatest value in separation of the fluorescent materials were $n$-butanol ( $50 \mathrm{vol}$.) + glacial acetic acid (10 vol.) + water (40 vol.) and $n$-butanol (40 vol.) +ethanol (40 vol.) + ammonia solution sp.gr. $0 \cdot 880$ (4 vol.) + water (16 vol.). The butanol was rendered non-fluorescent by shaking with norite and re-distilling, and butanol/ acetic acid/water mixtures were utilized after $48 \mathrm{hr}$. equilibration at room temperature. When necessary chromatograms were sprayed with $0.1 \%$ ninhydrin in purified $n$-butanol.

Paper electrophoresis. The method of Flynn \& de Mayo (1951) was used, with

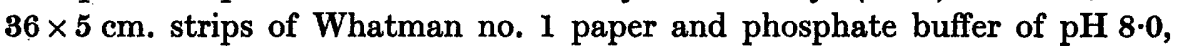
$\mu=0 \cdot 1$. Voltage applied $126 \mathrm{v}$. and time of running $11 \mathrm{hr}$.

Spot tests. Aldehydes were detected by the methods of Davidson \& Gregory (1932) and Bland (1949); phenolic groups according to Davidson \& Gregory (1932) and by spraying chromatograms with diazotized $p$-chloraniline ( 2 vol. of $0.02 \mathrm{M}$ - $p$-chloraniline in $0.25 \mathrm{~N}-\mathrm{HCl}+1$ vol. of $0.5 \% \mathrm{NaNO}_{2}$ ).

Ultraviolet absorption spectra. These were determined using a Unicam Spectrophotometer with a $2 \mathrm{~mm}$. cell and water as the blank.

\section{RESULTS}

\section{Chromatography of the fluorescent materials}

In order to examine for the possible presence of fluorescent materials in normal hair a sample of uninfected hair from children was extracted in the same way as for infected hair. The amount of hair used was approximately 50 times that of the infected hair and the resultant extract showed considerable fluorescence of a darker blue than that seen in infected hair extracts. A chromatogram developed with $n$-butanol/glacial acetic acid/water mixture showed: $(a)$ some faint fluorescence which had not moved from the starting point; $(b)$ a dark blue slowly moving fluorescent spot; $(c)$ a faint blue fast moving spot.

Extracts of hair infected with any of the three fungi contained considerable amounts of material which reacted with ninhydrin, and an attempt was made to find solvent mixtures which would give good separation of the fluorescent materials and at the same time separation from ninhydrin-reacting substances. The butanol/acetic acid/water and butanol/ethanol/ammonia/water mixtures proved the most satisfactory of the many tried. 
Extracts from hair infected by each fungus were examined separately and compared. Chromatograms of all three extracts showed a main brilliant greenish blue fluorescent spot, which was not present in the extract of normal hair, moving in each case and with all solvent mixtures used to an identical position (Pl. 1, fig. $1 a-c$; fig. $2 a, b$ ). A dark blue fluorescent spot was also clearly seen a little below this in extracts of hair infected by Microsporon canis and Trichophyton schoenleini (Pl. 1, fig. $1 d-f$; fig. $2 c$ ) but was only very faintly visible in chromatograms of the Microsporon audouini extracts. This latter blue spot occurred in the same position as the slow moving dark blue spot present in the chromatogram from normal hair. A faint light-blue fluorescent spot was present in extracts from hair infected by $M$. canis and showed much more strongly in that from the Trichophyton schoenleini infection (Pl. 1, fig. $1 \mathrm{~g}-i)$. This spot was not found in normal hair extract and has not been seen in extracts from hair infected by Microsporon audouini. Additional fluorescent spots, yellow and light blue, were present only in the extract from Trichophyton schoenleini infected hair (Pl. 1, fig. $1 j-m$ ).

Pl. 1, fig. 2, shows the result of spraying with ninhydrin a chromatogram prepared from the extracts of Microsporon canis and $M$. audouini infected hair and indicates the considerable separation which can be achieved of the fluorescent materials from ninhydrin-reacting material. This fact was utilized in the purification of the main green fluorescent material from such extracts. The initial hair extract was run on a chromatogram on Whatman no. 3 paper with the $n$-butanol/acetic acid/water mixture, the main fluorescent band eluted with $1 \cdot 17 \mathrm{~N}-\mathrm{NH}_{3}$ by shaking at room temperature for 20 min. followed by immediate concentration in vacuo to low volume and the procedure repeated using the $n$-butanol/ethanol/ammonia/water mixture. The final eluate was not entirely free from ninhydrin-reacting material.

Attempts to purify the material by column chromatography using kieselguhr, Decalso and Whatman powdered filter-paper showed no adsorption of the fluorescent material. When the fluorescent material was dissolved in $60 \%$ $(\mathrm{v} / \mathrm{v})$ isopropanol and added to a column of alumina (British Drug Houses Ltd. alumina for chromatographic analysis) the fluorescent substance was strongly adsorbed at the top of the column and resisted all attempts at elution. Mixtures of kieselguhr and alumina in differing proportions yielded the same result.

Paper electrophoresis. Further evidence for the identity of the green and dark-blue fluorescent spots in the extracts from Microsporon canis and Trichophyton schoenleini infected hair was obtained by paper electrophoresis (Pl. 2, fig. 3d-g), but the light-blue fluorescent spot seen in chromatograms of these extracts was only seen in the extract from the $T$. schoenleini infection (Pl. 2, fig. 3b). All three materials moved towards the anode indicating that they were negatively charged at $\mathrm{pH} 8 \cdot 0$.

The additional fluorescent materials found in chromatograms from the T. schoenleini infection are also seen. (Pl. 2, fig. $3 a, c)$; the blue fluorescent spot moved a short distance towards the anode, while the yellow spot moved a very short distance from the starting-point towards the cathode, this move- 
ment being almost certainly due to the electro-endosmosis occurring during the experiment.

Ultraviolet absorption spectra. The spectra of a number of the purified extracts from Microsporon canis and $M$. audouini infected hair were examined in the range of $\mathrm{pH} \mathrm{6-7.} \mathrm{All} \mathrm{showed} \mathrm{strong} \mathrm{end} \mathrm{absorption} \mathrm{but} \mathrm{no} \mathrm{constant}$ characteristic curve was obtained, as also reported by Robinson et al. (1953) for extracts of $M$. audouini infected hair. Below $300 \mathrm{~m} \mu$ the results varied considerably from one extract to another; with extracts from smaller amounts of fluorescent hair no specific absorption between 300 and $400 \mathrm{~m} \mu$. was found. With the more concentrated extracts there was a small amount of light absorption between 310 and $340 \mathrm{~m} \mu$. not seen in weaker solutions, although these were still strongly fluorescent. It seems probable that the fluorescent substance has not yet been obtained sufficiently concentrated to give a characteristic spectrum.

\section{Chemical properties}

Extracts of the principal greenish blue fluorescent material from Microsporon canis and $\boldsymbol{M}$. audouini infected hair, purified by chromatography as described above and concentrated finally to a volume of $c .1 .0 \mathrm{ml}$., were subjected to a number of spot tests. The final concentrate was pale yellow in colour, slightly opalescent, exhibited a vivid greenish blue fluorescence in ultraviolet light, and on evaporation to dryness left a just discernible yellowish residue. The tests for aldehyde and phenolic groups used by Davidson \& Gregory (1932) both yielded positive results. A sample, $0.1 \mathrm{ml}$,, of the concentrate was dried drop by drop on filter-paper and sprayed with 2:4-dinitrophenylhydrazine but no colour change was discernible. Treatment of $0.2 \mathrm{ml}$. of concentrate with this reagent and warming for $30 \mathrm{~min}$. at $37^{\circ}$ led to the separation of a few crystals, visible microscopically, but insufficient for characterization. The presence of a phenolic substance was confirmed by treatment with diazotized $p$-chloraniline. The aldehyde and phenolic group tests on the crude extract from Trichophyton schoenleini infected hair were also positive. Spraying of chromatograms with diazotized $p$-chloraniline followed by exposure to ammonia vapour, however, showed the phenolic substance to be associated with the dark blue fluorescent spot and not with the main fluorescent material. This finding was confirmed by spraying the results of paper electrophoresis with the same reagent; no colour with the phenolic reagent was given by the main fluorescent material. The presence of this dark blue fluorescent substance in the final eluate from chromatography was due to incomplete separation of the two fluorescent materials on the Whatman no. 3 paper. Examination of the crude extract of the $T$. schoenleini infected hair and of chromatograms developed from it gave the same results.

The fluorescence of extracts from hair infected with any of the three fungi was quenched immediately by the addition of bromine water or by sodium hydrosulphite, being regenerated in the latter case by shaking the solution in air, thus confirming the finding of Robinson et al. (1953) with a fluorescent 
extract of Microsporon audouini infected hair. There was no change in the fluorescence on treatment with hydrogen peroxide or sodium sulphite. No colour was given with ferric chloride.

\section{DISCUSSION}

Whilst growing as parasites in the hair root, Microsporon canis, M. audouini and Trichophyton schoenleini all cause the production of fluorescent substances in the cortex of the hair. They do not, however, produce these substances in artificial culture, so that the supply of fluorescent material is necessarily very limited. A number of attempts have been made to infect animals experimentally with Microsporon canis, but only small areas of infection have been obtained in guinea-pigs and all attempts to infect rats and mice have failed. The initial extract from the infected hair is a complex mixture of breakdown products of keratin and, presumably, other metabolic products from the invading fungus. Separation from ninhydrin-reacting substances by the techniques described was not complete and it is still an open question as to whether the main fluorescent substance is a peptide; Davidson \& Gregory (1932) reported no ninhydrin-reacting material in their extracts, while Robinson et al. (1953) concluded from infra-red analysis of their extract from M. audouini infected hair, that the material resembled a peptide. They produced, however, no evidence that their procedure for purification had separated the fluorescent substance from all the other materials and in particular from the products of keratin breakdown known to be present in the initial aqueous extracts of infected hair, or from the fluorescent substances now shown to be present in normal hair. In view of the small amount of material available chemical properties can only be examined by spot tests, and every endeavour has been made to relate these results to areas of fluorescence as shown by paper chromatography, and only results which have been consistently positive on a series of extracts over the past 4 years have been recorded.

We wish to thank Dr J. T. Ingram for permission to obtain infected hair from his patients, Dr J. Walker and Dr C. J. La Touche for identifying cultures from many of the patients, Mr T. J. Bowen for advice on paper electrophoresis techniques and Mr C. N. England for carrying out the photography.

\section{REFERENCES}

BLAND, D. E. (1949). Separation of vanillin and syringaldehyde by paper partition chromatography. Nature, Lond. 164, 1093.

Consden, R., Gordon, A. H. \& Martin, A. J. P. (1944). Qualitative analysis of proteins: partition chromatographic method using paper. Biochem. J. 38, 224.

Davidson, A. M. \& Gregory, P. H. (1932). Note on an investigation with the fluorescence of hairs infected by certain fungi. Canad. J. Res. 7, 378.

Felsher, Z. (1949). Observations on the fluorescent material in hairs infected by Microsporon in tinea capitis. J. invest. Derm. 12, 139.

FlynN, F. V. \& DE Mayo, P. (1951). Micro-electrophoresis of protein on filter paper. Lancet, ii, 235. 
Journal of General Microbiology, Vol. 11, No. 3

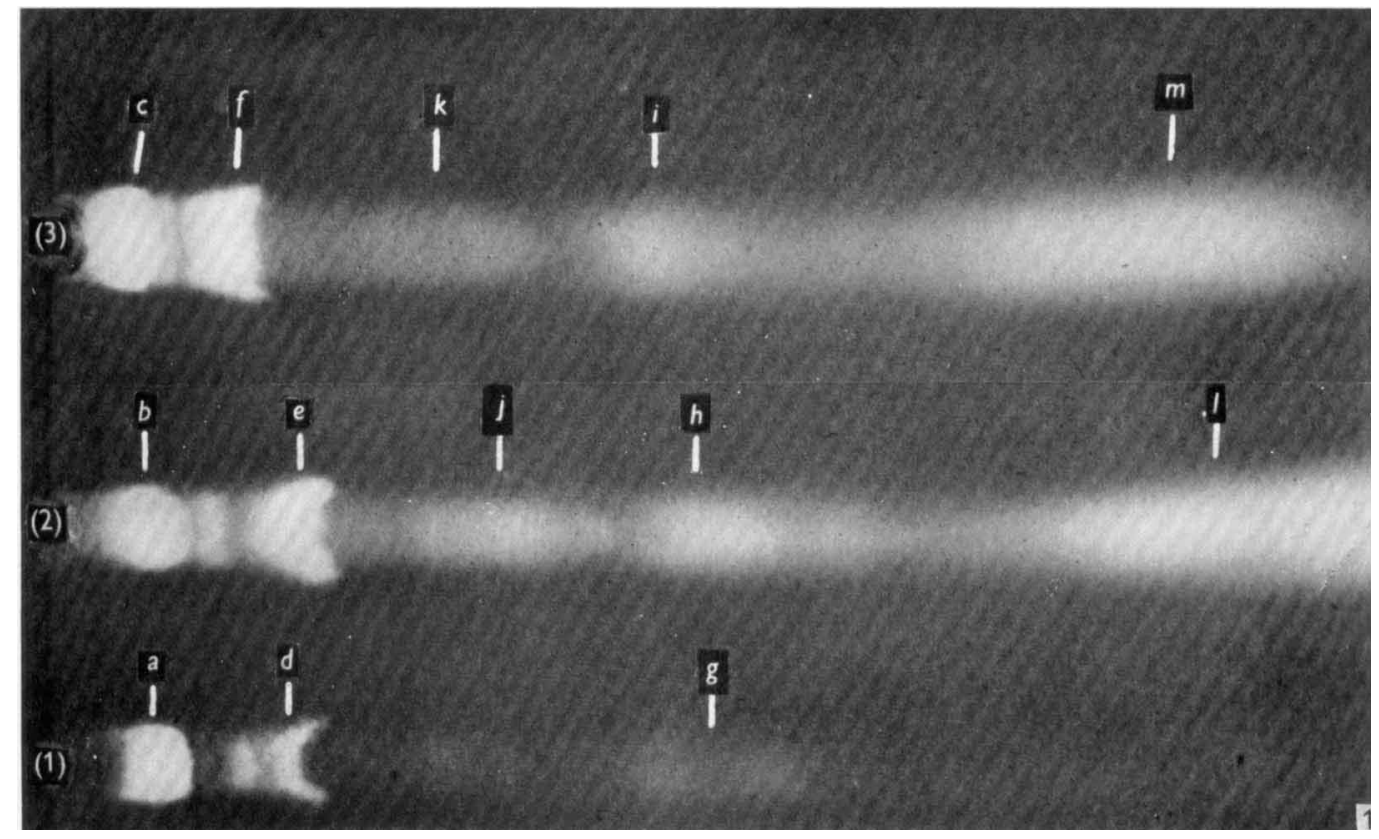

Fig. 1

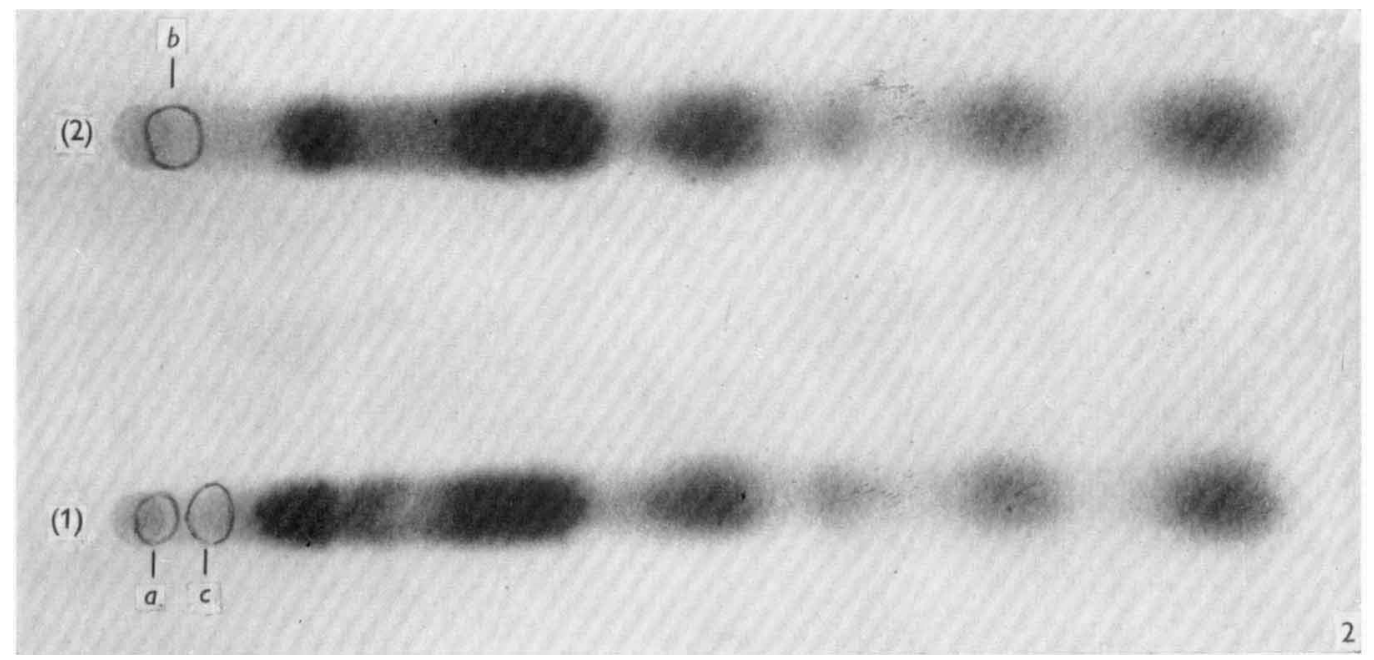

Fig. 2

F. W. Chattaway and A. J. E. Barlow-Fluorescence of dematophytes $i n$ vivo. Piate 1 
Journal of General Microbiology, Vol. 11, No. 3

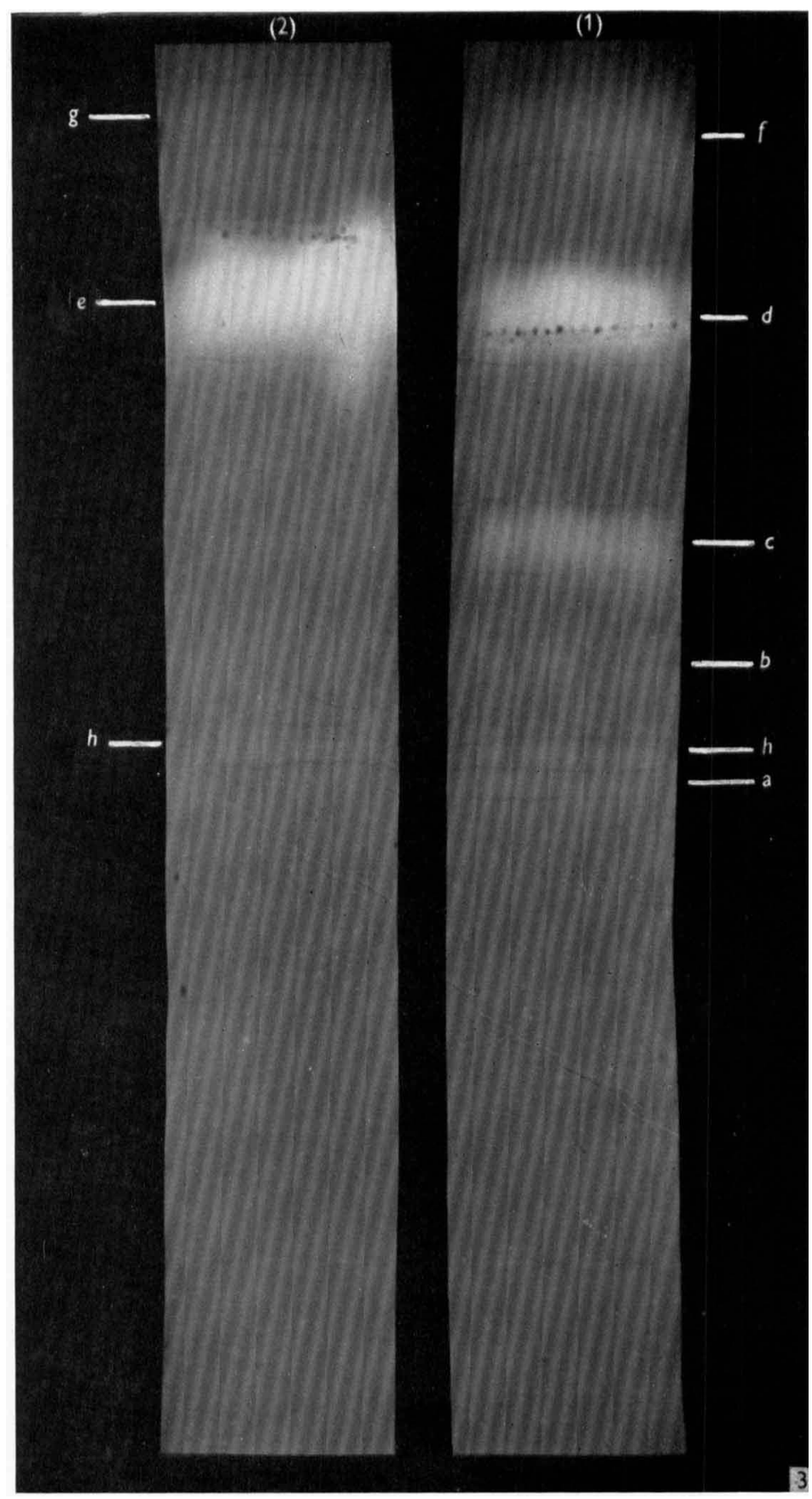

F. W. Chattayay and A. J. E. Barlow-Fluorescence of dermatophytes in vivo. Plate 2 


\section{Fluorescence of dermatophytes in vivo}

Margarot, J. \& Devìze, P. (1925). Aspect de quelques dermatoses en lumière ultraparaviolette: note préliminaire. Bull. Soc. Sci. méd. biol. Montpellier, 6, 375.

Robinson, Jun., H. M., Figge, F. H. J. \& Bereston, E. S. (1953). Fluorescence of Microsporon audouini-infected hair. Arch. Derm. Syph. 68, 129.

\section{EXPLANATION OF PLATES}

\section{Plate 1}

Fig. 1. Chromatogram developed with $n$-butanol/glacial acetic acid/water on Whatman no. 3 paper of crude extracts of hair infected with (1) Microsporon canis, (2) Trichophyton schoenleini, (3) a mixture of extracts (1) and (2). Areas of fluorescence: $(a),(b)$, $(c)$ brilliant greenish blue; $(d),(e),(f)$, dark blue also seen in extracts of normal hair; $(g),(h),(i)$ light blue; $(j),(k)$ yellow, $(l),(m)$ light blue. Photographed in ultraviolet light using Ilford S.R. panchromatic plate with an Osira black lamp, MBW/V, at a distance of $1 \mathrm{ft}$. with exposure time 10 sec. at $\mathrm{f} .16$.

Fig. 2. Chromatogram developed with $n$-butanol/ethanol/ammonia solution sp.gr. 0.880/ water on Whatman no. 3 paper of crude extracts of hair infected with (1) Microsporon canis, (2) M. audouini. Spots $(a)$ and $(b)$ are areas of brilliant greenish blue fluorescence; spot $(c)$ an area of dark blue fluorescence, also seen in extracts of normal hair. Dark areas represent colours produced on spraying with $1 \%$ ninhydrin in $n$-butanol.

\section{Plate 2}

Fig. 3. Paper electrophoresis of crude extracts of hair infected with (1) Trichophyton schoenleini, (2) Microsporon canis. Areas of fluorescence: $(a)$ yellow; $(b),(c)$ light blue; $(d),(e)$ bright greenish blue; $(f)$, (g) dark blue. Spot $(h)$ was the starting-point. Photographed in ultraviolet light using Ilford S.R. panchromatic plate with two Osira black lamps at a distance of $1 \mathrm{ft}$. with exposure time $16 \mathrm{sec}$. at $\mathrm{f} .8$.

(Received 13 July 1954) 